\title{
CTAB 对多级孔分子篮合成及孔道层次结构影响
}

\author{
李红现 ${ }^{1,2}$, 周孝德 ${ }^{1}$, 张建民 ${ }^{2}$, 杨 靖 $^{2}$, 王发良 ${ }^{2}$, 王向阳 ${ }^{2}$
}

(1. 西安理工大学 水利水电学院, 西安 710048; 2. 西安工程大学 环境与化学工程学院, 西安 710048)

摘 要: 以预处理后的凹凸棒石(Si-ATP)为前驱体, 采用双模板剂一步法合成了多级孔分子篮, 研究了结构导向剂 十六烷基三甲基溴化铵(CTAB)用量对分子篮孔道层次结构和种类的影响。结果表明：当 CTAB 用量为 $0.01 ~ 0.03 \mathrm{~g}$ 或 0.05 0.07 g 时, 孔道层次结构因子(HF)与 CTAB 用量呈线性相关; XRD 结果显示, 当 CTAB 用量为 $0.03 \sim 0.05 \mathrm{~g}$ 时, 分子篎从 ZSM-5 型向(H)ZSM-11 型转变, 这说明在利用此方法制备多级孔分子篎时, 可通过调变 CTAB 用量定向 调控分子篮微介孔比例。CTAB 用量为 $0.05 \mathrm{~g}$, 合成的 $(\mathrm{H}) Z \mathrm{ZSM}-11$ 分子篮比表面积 $\left(S_{\mathrm{BET}}\right)$ 为 $432.02 \mathrm{~m}^{2} / \mathrm{g}$, 总孔体积 $\left(V_{\mathrm{tot}}\right)$ 为 $0.40 \mathrm{~cm}^{3} / \mathrm{g}$, 亚甲基蓝(MB)的吸附容量为 $366.45 \mathrm{mg} / \mathrm{g}$, 说明 CTAB 导向构筑的介孔孔道有利于吸附大分子物 质。

关 键 词: Si-ATP; CTAB; 多级孔分子篮; 孔道层次结构因子

中图分类号: TQ426 文献标识码: A

\section{CTAB on Synthesis and Pore Structure of Hierarchical Zeolite}

\author{
LI Hong-Ji ${ }^{1,2}$, ZHOU Xiao-De ${ }^{1}$, ZHANG Jian-Min ${ }^{2}$, YANG Jing ${ }^{2}$, WANG Fa-Liang ${ }^{2}$, WANG Xiang-Yang ${ }^{2}$ \\ (1. Faculty of Water Resources and Hydraulic Power, Xi'an University of Technology, Xi'an 710048, China; \\ 2. School of Environment and Chemistry Engineering, Xi'an Polytechnic University, Xi'an 710048, China)
}

\begin{abstract}
Hierarchical zeolite was successfully synthesized with pretreated attapulgite (Si-ATP) by dual-template in one pot. Influence of the amount of structure-directing agent, hexadecyl trimethyl ammonium bromide (CTAB), on hierarchical pore structure and crystal morphology was systematically investigated. The results showed that the hierarchy factor (HF) had a good linear dependence on CTAB amount when it was between 0.01 and $0.03 \mathrm{~g}$ or 0.05 and 0.07 g. XRD revealed that the crystal morphology transferred from ZSM-5 to (H)ZSM-11 when CTAB amount ranged from 0.03 to $0.05 \mathrm{~g}$. These results indicated that the ratio of micropore and mesopore could be controlled by CTAB amount when preparing hierarchical zeolites by this method. (H)ZSM-11 zeolites, prepared with $0.05 \mathrm{~g}$ of CTAB, exhibited a high specific surface area $\left(S_{\mathrm{BET}}=432.02 \mathrm{~m}^{2} / \mathrm{g}\right)$, a large total volume $\left(V_{\mathrm{tot}}=0.40 \mathrm{~cm}^{3} / \mathrm{g}\right)$, and an adsorption capacity of methylene blue (MB) $(366.45 \mathrm{mg} / \mathrm{g})$. Therefore, the mesoporous pore built by CTAB is beneficial for adsorbing of macromolecules.
\end{abstract}

Key words: Si-ATP; CTAB; hierarchical zeolite; hierarchical factor

近年来, 多级孔分子笁因兼具微孔分子篮良好 热稳定性和介孔分子笁较强分子扩散性的特点而备
受广大研究学者亲睐。目前, 合成多级孔分子筛的 方法主要有两种: 一种是模板法, 即利用模板剂构

收稿日期：2017-07-17; 收到修改稿日期：2017-10-26

基金项目: 国家自然科学基金(21573171); 陕西省重点研发计划(2017GY-121); 陕西省教育厅专项科研计划(17JK0327); 陕 西省大学生创新创业计划 $(1715,1731)$

National Natural Science Foundation of China (21573171); Shaanxi Province Key Research and Development Plan (2017GY-121); Shaanxi Provincial Natural Science Foundation (17JK0327); Research and Innovation Training Project for Graduate in General Universities of Shaanxi Province $(1715,1731)$

作者简介: 李红玑(1986-), 女, 讲师. E-mail: lhj861106@126.com 
筑微、介孔孔道形成多级孔 ${ }^{[1-3]}$, 此方法简单易行、 应用普遍; 另一种是后处理法 ${ }^{[4]}$, 即先水热合成微 孔分子篮, 再脱除晶体骨架中硅和铝形成介孔孔道 ${ }^{[-7]}$, 以扩大微孔而构建介孔，但这种方法降低了分子篮 稳定性, 因此在应用中受限。合成采用的硅源一般 为有机硅(正硅酸乙酯)、硅胶、固体二氧化硅和无 定形硅, 通常这些物质只提供化合价态的硅作为合 成原料, 而不具有与分子篮类似的孔道层次结构。 凹凸棒石(Attapulgite, ATP $)^{[8-10]}$ 是一种天然纳米层 状硅酸盐材料, 富含两层硅氧四面体, 横断面具有 $0.37 \mathrm{~nm} \times 0.63 \mathrm{~nm}$ 孔道, 通过碱焙烧、酸活化处理后, 可以保留原有纳米孔道, 在结构导向剂 CTAB 作用 下重新构筑孔道层次结构, 利用硅氧四面体作为基 本骨架定向合成某种多级孔分子篮。

ZHAO 等 ${ }^{[11]}$ 利用碱溶 ATP 作为硅源, 与铝酸钠 在 $90^{\circ} \mathrm{C}$ 水热晶化 $6 \mathrm{~h}$, 成功制备 $4 \mathrm{~A}$ 分子篮, 说明 ATP 为硅源合成分子篎具备可行性。SHAO 等 ${ }^{[12]}$ 在 $\mathrm{H}_{3} \mathrm{PO}_{4}$ 和四乙基氢氧化铵(TEA)作用下, 利用预处 理 ATP 成功合成出 Me SAPO-5 分子篎, 进一步证明 二者之间可相互转化。ZHENG 等 ${ }^{[13]}$ 在高岭土微球 中加入硅铝凝胶作为硬模板, 通过水热晶化成功合 成 $\mathrm{NaY}$ 分子笁为基质的多孔复合材料, 其介孔孔容 为 $0.22 \mathrm{~cm}^{3} / \mathrm{g}$, 介孔比表面积为 $98.90 \mathrm{~m}^{2} / \mathrm{g}$, 为粘土 类硅酸盐矿物作为前驱体合成多级孔分子篮提供了 有利证据。ZHOU 等 ${ }^{[14]}$ 利用酸活化 ATP 作为硅源, 合成出介孔孔容为 $0.08 \mathrm{~cm}^{3} / \mathrm{g}$ 的多级孔 Fe/Ti-ZSM-5 分子篮。但利用预处理 ATP 作为分子篮前驱体, 考 察结构导向剂十六烷基三甲基澳化铵(CTAB)对合 成的多级孔分子篮孔道层次结构和种类影响的研究 鲜有报道。

本工作以碱焙烧复合酸活化后的凹凸棒石 (Si-ATP)为前驱体, 采用双模板剂一步水热晶化法, 定向合成具有微介孔的多级孔分子篮。通过 FT-IR、 XRD、SEM 和 $\mathrm{N}_{2}$ 吸附-脱附仪等表征分子篮的组 分、形貌及孔道结构, 分析产物孔道和种类变化, 揭 示孔道层次结构因子 HF 与 CTAB 用量之间的关系, 研究 CTAB 用量对多级孔分子篮孔道层次结构及种 类的影响。

\section{1 实验方法}

\section{1 多级孔分子篮合成}

将 ATP 与 $\mathrm{NaOH}$ 按质量比 $1: 1$ 放入马弗炉, 以 $10^{\circ} \mathrm{C} / \mathrm{min}$ 速率升温至 $550^{\circ} \mathrm{C}$ 焙烧 $2 \mathrm{~h}$, 冷却至室温, 用 $1.00 \mathrm{~mol} / \mathrm{L} \mathrm{NaCl}$ 溶液洗涤干燥。称取一定量焙烧 产物, 按固液比 $1: 8$ 加入 $9 \%$ 盐酸溶液, 置于 $180^{\circ} \mathrm{C}$
烘箱中水热 $12 \mathrm{~h}$, 取出离心洗涤干燥, 即得分子篮 前驱体记为 Si-ATP。

准确称取 $0.011 \mathrm{~g} \mathrm{NaAlO}_{2}$ 和 $7.70 \mathrm{~mL}$ 四丙基氢 氧化铵(TPAOH), 加入一定量 CTAB, 置于 $5.20 \mathrm{~mL}$ 去离子水中均匀溶解, 称取 $0.80 \mathrm{~g} \mathrm{Si-ATP}$ 加入其中, 室温环境下摚拌 $4 \mathrm{~h}$ 得初始凝胶; 将其移入 $25 \mathrm{~mL}$ 水热晶化釜中, 在 $180^{\circ} \mathrm{C}$ 恒温烘箱中晶化反应 $4 \mathrm{~h}$, 待冷却后对产物进行抽滤、洗涤、干燥, $550^{\circ} \mathrm{C}$ 马弗 炉中锻烧 $5 \mathrm{~h}$, 去除模板剂后获得最终反应产物, 记 为 $\mathrm{CTAB} n$ 。 $n$ 即 $\mathrm{CTAB}$ 投加量, 分别为 $0.01 、 0.02$ 、 $0.03 、 0.04 、 0.05 、 0.06 、 0.07 \mathrm{~g}$ 。

\section{2 亚甲基蓝水样制备}

准确称取 $50.00 \mathrm{mg}$ 亚甲基蓝染料, 溶于 $1.00 \mathrm{~L}$ 去离子水中, 定容至 $50.00 \mathrm{mg} / \mathrm{L}$ 。

\section{3 样品表征}

使用日本岛津公司 IRPrestige-21 傅立叶变换 红外光谱仪(FT-IR)对样品表面官能团进行分析; 利 用日本岛津公司 X 射线衍射分析仪(XRD)分析样品 的晶型和种类; 采用英国 FEI Quanta-450-FEG 扫描 电镜(SEM)对样品表面形貌进行观察; 采用美国 Micromeritics ASAP 2020 PlusHD88 型物理吸附仪 测定样品 $\mathrm{N}_{2}$ 吸脱附等温线, 总孔容由相对压力 $\left(p / p_{0}\right)$ 为 0.95 时的氮吸附量换算成液氮体积得到, 采用 BET 法计算比表面积, 孔面积由 $t$ 图法获得, 由 BJH 法计算孔径参数。引入孔道层次结构因子 $(\mathrm{HF})$ 评价 分子篮的孔道层次结构 ${ }^{[15-16]}$, 公式如下:

$$
\mathrm{HF}=\left(V_{\text {micro }} / V_{\text {tot }}\right) \times\left(S_{\text {mes }} / S_{\text {BET }}\right)
$$

式中, $V_{\text {micro }}$ 为微孔体积, $V_{\text {tot }}$ 为总孔体积; $S_{\text {mes }}$ 为孔外 表面积, $S_{\mathrm{BET}}$ 为孔总比表面积。

\section{2 结果与讨论}

\section{1 红外光谱分析}

图 1 为 ATP、Si-ATP 和 CTAB0.05 样品的红外 谱图, 产物在 1631.83、954.01、551.71 $\mathrm{cm}^{-1}$ 处出现 了新峰, 说明产生了新结构及官能团; $1631.83 \mathrm{~cm}^{-1}$ 处 为 $\delta(\mathrm{H}-\mathrm{O}-\mathrm{H})$ 键说明 Si-ATP 存在结构水; $954.01 \mathrm{~cm}^{-1}$ 处为 $\mathrm{Si}-\mathrm{O}$ 键不对称伸缩振动峰, 主要是活化导致 结构非对称性变化并形成 $\mathrm{T}-\mathrm{O}$ 四面体 $\mathrm{Si}-\mathrm{O}_{4}$ 在 $\mathrm{i}$ 轴 方向的伸缩振动, 说明 Si-ATP 活化后导致层链状结 构破坏, 从而形成分子篮结构中常见的 $\mathrm{Si}-\mathrm{O}_{4}$ 自由 基团; $551.71 \mathrm{~cm}^{-1}$ 处一般为(H)ZSM-11 分子篮骨架 中 $d 5 r$ 特征峰, 属于双环孔道结构, 进一步说明 Si-ATP 与分子篮具有类似结构。特征峰位置发生四 处移动, 分别是 $\mathrm{O}-\mathrm{Si}-\mathrm{O}$ 非对称键伸缩振动峰从 $1080.08 \mathrm{~cm}^{-1}$ 移至 $1093.56 \mathrm{~cm}^{-1} ; \delta(\mathrm{Si}-\mathrm{O}-\mathrm{Si})$ 振动峰 从 $781.23 \mathrm{~cm}^{-1}$ 移至 $798.04 \mathrm{~cm}^{-1}$; O-Si-O弯曲振动峰 


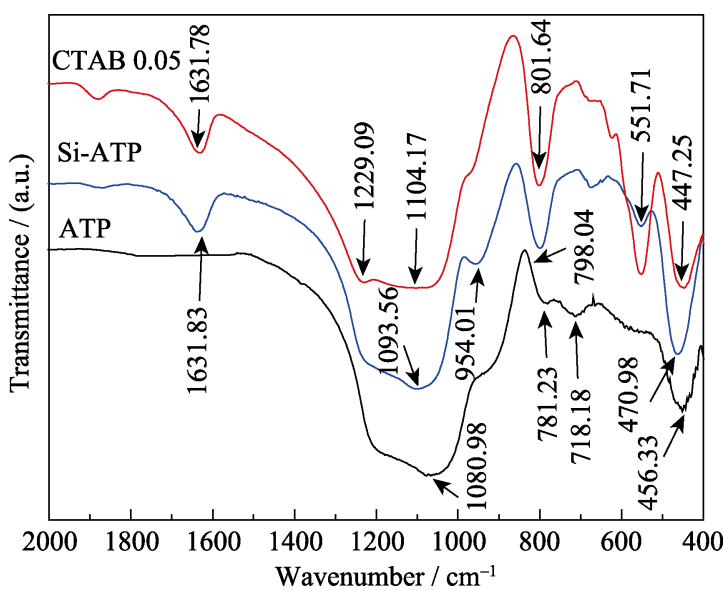

图 1 ATP、Si-ATP 和 CTAB0.05 晶化产物的 FT-IR 谱图 Fig. 1 Infrared spectra of ATP, Si-ATP and crystalline product with $0.05 \mathrm{~g}$ CTAB

从 $456.33 \mathrm{~cm}^{-1}$ 移至 $470.98 \mathrm{~cm}^{-1}$, 振动峰均朝高波数 方向偏移，说明其键强增强; $\mathrm{Si}-\mathrm{O}$ 键对称伸缩振动 峰从 $718.18 \mathrm{~cm}^{-1}$ 移至 $668.79 \mathrm{~cm}^{-1}$, 振动峰朝低波数 方向偏移, 则其键强减弱。上述结果说明 Si-ATP 整体骨架结构发生不对称和非稳定转化, 降低反应 所需活化能。

\section{$2.2 \mathrm{XRD}$ 分析}

图 2 为 ATP、Si-ATP 及不同 CTAB 用量下晶化 产物的 XRD 图谱, 从该图可以看出, 分子篎前驱体 Si-ATP 的特征峰强度较 ATP 的特征峰明显减弱, 呈 非晶态, 产物 $2 \theta=16.13^{\circ} 、 27.16^{\circ}$ 处的特征峰与 $\mathrm{Na}$ 型 $4 \mathrm{~A}$ 分子篮特征峰相近; 当 CTAB 用量为 $0 \mathrm{~g}$, 产 物 $2 \theta=21.55^{\circ} 、 27.08^{\circ}$ 和 $50.65^{\circ}$ 处出现的特征峰与 ATP 特征峰类似, 说明未形成分子篮结构; 当 $\mathrm{CTAB}$ 用量为 $0.01 \mathrm{~g}$ 时, 产物 $2 \theta=23.08^{\circ} 、 23.32^{\circ}$ 、 $23.95^{\circ} 、 24.41^{\circ}$ 和 $24.78^{\circ}$ 处形成明显的 ZSM-5 分子 筛特征峰; CTAB 用量增至 $0.04 \mathrm{~g}$, 产物 $2 \theta=22^{\circ} \sim 24^{\circ}$ 范围内五指峰的分峰减弱, 同时 $2 \theta=44^{\circ} \sim 45^{\circ}$ 处逐渐 形成分裂峰, 说明产物从 ZSM-5 型向(H)ZSM-11 型 转化 ${ }^{[15]}$; CTAB 用量为 $0.05 \sim 0.07 \mathrm{~g}$ 时, 产物仅有 $2 \theta=23.03^{\circ} 、 23.86^{\circ}$ 和 $24.36^{\circ}$ 三个特征峰, 说明此样品 为 $(\mathrm{H}) \mathrm{ZSM}-11$ 分子篮。

\subsection{SEM 分析}

图 3 为晶化产物的表面形貌, 可以看到 CTAB 用量为 $0.01 \sim 0.03 \mathrm{~g}$, 颗粒呈典型 ZSM-5 分子篎的六 面体形状, 且尺寸从 $350 \mathrm{~nm} \times 400 \mathrm{~nm}$ 增至 $680 \mathrm{~nm} \times$ $800 \mathrm{~nm}$, 说明 CTAB 可促使颗粒生长; 当 CTAB 用 量增至 $0.04 \mathrm{~g}$, 产物中出现六面体和椭球状混晶, 说明产物发生了晶体形状变化, 结合 XRD 结果可知, 产物由 ZSM-5 型向 $(H) Z S M-11$ 型转变; 当 CTAB 增 至 $0.05 \mathrm{~g}$ 时, 颗粒呈椭球形, 是典型的 $(\mathrm{H}) \mathrm{ZSM}-11$

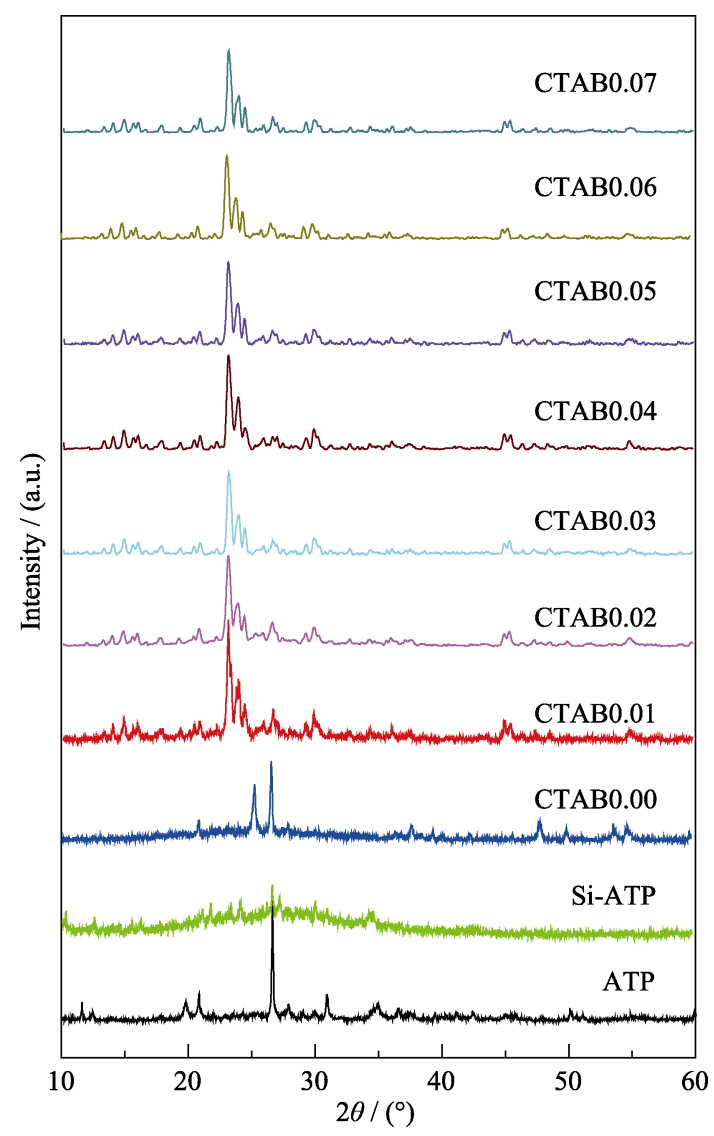

图 2 ATP、Si-ATP 及不同 CTAB 用量条件下晶化产物的 $\mathrm{XRD}$ 图谱

Fig. 2 XRD patterns of ATP, Si-ATP and crystalline products with different CTAB additions

分子篮形状。结合 FT-IR 分析, Si-ATP 分子篮前驱 体具有 $\mathrm{Si}-\mathrm{O}_{4}$ 官能团和双环孔道结构, 结构导向剂 CTAB 用量为 $0.04 \mathrm{~g}$ 时, 促使前驱体环状结构链长 增长, 从 ZSM-5 分子篮的五元环结构变为 $(\mathrm{H}) \mathrm{ZSM}-11$ 分子篮的十元环结构 ${ }^{[17]}$, 并抑制了晶面在 $c$ 轴方向 上的生长, 导致颗粒从六面体转化为椭球型。

\section{4 比表面积及孔径分析}

图 4 的曲线显示, 根据 IUPAC 划分, CTAB 用量 为 $0.01 \sim 0.04 \mathrm{~g}$ 时, 晶化产物的吸附-脱附曲线符合 I 型等温线 ${ }^{[18]}$, 相应于朗格缪尔单层窄孔可逆吸附, 说明晶化产物孔道以微孔为主; CTAB 用量为 0.05 $0.06 \mathrm{~g}$ 时分子篎吸附-脱附曲线符合 IV 型等温线, 在 较低 $p / p_{0}$ 曲线凸向上, 呈现单分子层的饱和吸附量, 至较高 $p / p_{0}$ 时, 等温线迅速上升, 主要是因为吸附 质发生毛细管凝聚，由此发生脱附等温线与吸附等 温线不重合的滞后现象, 滞后环与孔的形状及其大 小有关。结合图 5 孔径分布分析, 孔径从 $2.00 \mathrm{~nm}$ 扩 展至 $4.50 \mathrm{~nm}$, 说明产物出现明显的介孔孔道, 与上 述 $\mathrm{N}_{2}$ 吸附-脱附曲线中滞后环出现一致, 由此证实 CTAB 作用于分子篮孔道结构变化。 

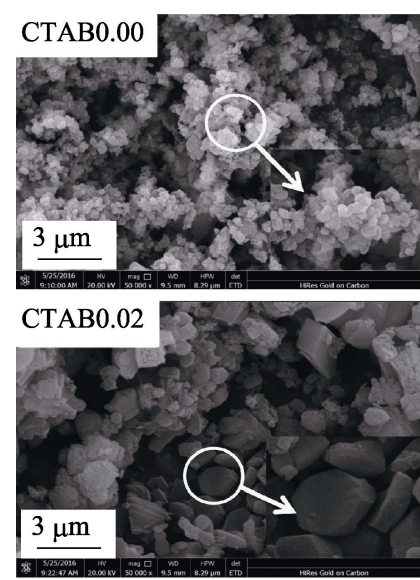

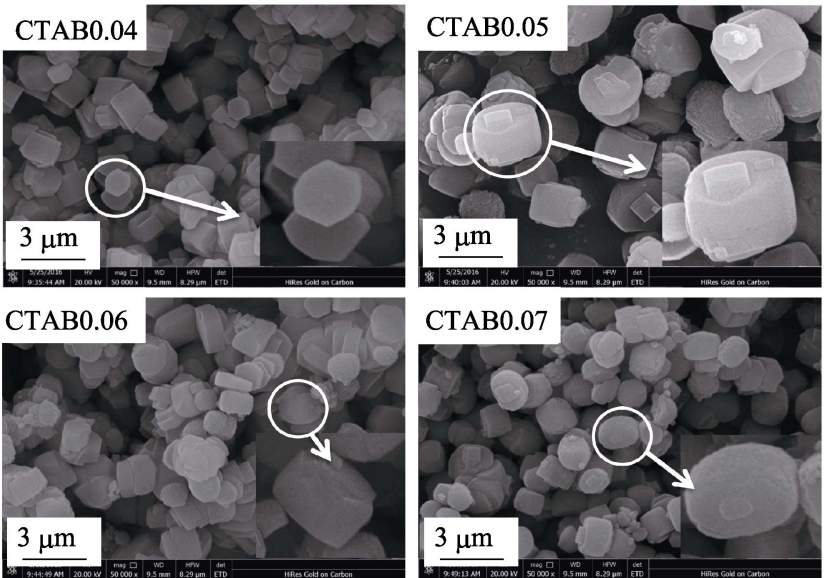

图 3 不同 CTAB 用量条件下晶化产物 SEM 照片

Fig. 3 SEM images of crystalline products with different CTAB additions

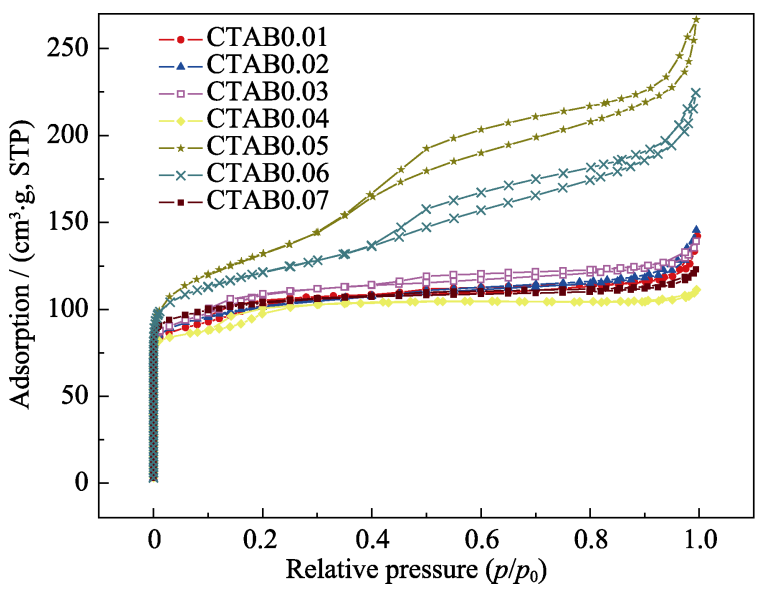

图 4 不同 CTAB 用量条件下晶化产物 $\mathrm{N}_{2}$ 吸附一脱附曲线

Fig. $4 \mathrm{~N}_{2}$ adsorption-desorption isotherms of crystalline products with different CTAB additions

\section{5 孔道层次结构分析}

$\mathrm{CTAB}$ 用量对晶化产物比表面积 $\left(S_{\mathrm{BET}}\right)$ 、孔体积 $\left(V_{\mathrm{tot}}\right)$ 等参数的影响如表 1 所示, 孔道层次结构因子 HF 计算结果见图 6。CTAB 用量为 $0.01 \sim 0.04 \mathrm{~g}$, 分 子篮 $S_{\mathrm{BET}}$ 变化保持在 $(300 \pm 25) \mathrm{m}^{2} / \mathrm{g}, V_{\text {tot }}$ 为 $(0.20 \pm$ $0.04) \mathrm{cm}^{3} / \mathrm{g}$, 说明在少量结构导向剂 CTAB 作用下,

表 1 不同 CTAB 用量条件下晶化产物质构特性

Table 1 Textural properties of crystalline products with different CTAB additions

\begin{tabular}{cccccccc}
\hline Sample & $\begin{array}{c}S_{\text {BET }} / \\
\left(\mathrm{m}^{2} \cdot \mathrm{g}^{-1}\right)\end{array}$ & $\begin{array}{c}S_{\text {mid }} \\
\left(\mathrm{m}^{2} \cdot \mathrm{g}^{-1}\right)\end{array}$ & $\begin{array}{c}S_{\text {mes }}\left(\mathrm{m}^{2} \cdot \mathrm{g}^{-1}\right) \\
\left(\mathrm{cm}^{3} \cdot \mathrm{g}^{-1}\right)\end{array}\left(\mathrm{cm}^{3} \cdot \mathrm{g}^{-1}\right)\left(\mathrm{cm}^{3} \cdot \mathrm{g}^{-1}\right)$ \\
\hline CTAB0.01 324.73 & 132.34 & 192.39 & 0.20 & 0.07 & 0.13 & 0.21 \\
CTAB0.02 321.85 & 118.35 & 203.50 & 0.21 & 0.07 & 0.14 & 0.20 \\
CTAB0.03 301.06 & 177.49 & 123.57 & 0.21 & 0.10 & 0.11 & 0.21 \\
CTAB0.04 313.20 & 112.75 & 200.45 & 0.16 & 0.06 & 0.10 & 0.24 \\
CTAB0.05 432.02 & 165.65 & 266.37 & 0.40 & 0.08 & 0.32 & 0.12 \\
CTAB0.06 372.38 & 189.08 & 183.30 & 0.34 & 0.11 & 0.23 & 0.18 \\
CTAB0.07 300.28 & 212.81 & 87.47 & 0.19 & 0.12 & 0.07 & 0.16 \\
\hline
\end{tabular}

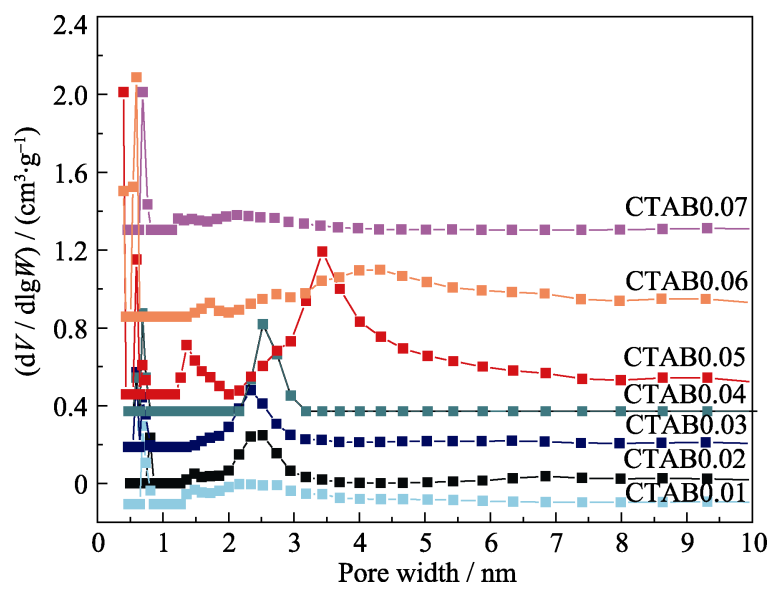

图 5 不同 CTAB 用量条件下晶化产物孔径分布图

Fig. 5 Pore size distribution of crystalline products with different CTAB additions

形成具有稳定的比表面积和孔道结构的多级孔分子 筛; CTAB 用量为 $0.05 \mathrm{~g}$ 时, $S_{\mathrm{BET}}$ 为 $432.02 \mathrm{~m}^{2} / \mathrm{g}, V_{\text {tot }}$ 为 $0.40 \mathrm{~cm}^{3} / \mathrm{g}$, 说明此时形成的分子篎孔道发达程 度最高。

结合 HF 计算结果, CTAB 用量在 0.01 0.03 g 范 围内, $S_{\mathrm{mes}} / S_{\mathrm{BET}}$ 与 CTAB 用量呈线性正相关, 相关系 数为 $0.9977, \mathrm{HF}$ 为 $(0.20 \pm 0.01)$, 说明 CTAB 用量越 高, $S_{\text {mes }}$ 越大, 导致介孔所占比例越大, 此时 CTAB 用量主要作用于介孔孔道构筑。CTAB 用量在 0.05 $0.07 \mathrm{~g}$ 范围内, $S_{\mathrm{mes}} / S_{\mathrm{BET}}$ 与 CTAB 用量呈线性负相关, 相关系数为 0.9773 , 说明 CTAB 用量继续增加将导 致介孔所占比例减小。CTAB 用量为 $0.03 \sim 0.05 \mathrm{~g}$ 时, HF 值呈无规律变化, 结合 XRD 和 SEM 分析可知, 此时 CTAB 主要使分子篎从五元环结构扩展至十元 环结构, 晶型从 ZSM-5 型转化为(H)ZSM-11 型, 并 导致晶体颗粒形状改变。孔道结构中, HF 值越小则 介孔越发达 ${ }^{[19]}$, CTAB0.05 的 HF 值为 0.12 , 说明此 时 CTAB 用量对介孔孔道构筑最有利。 


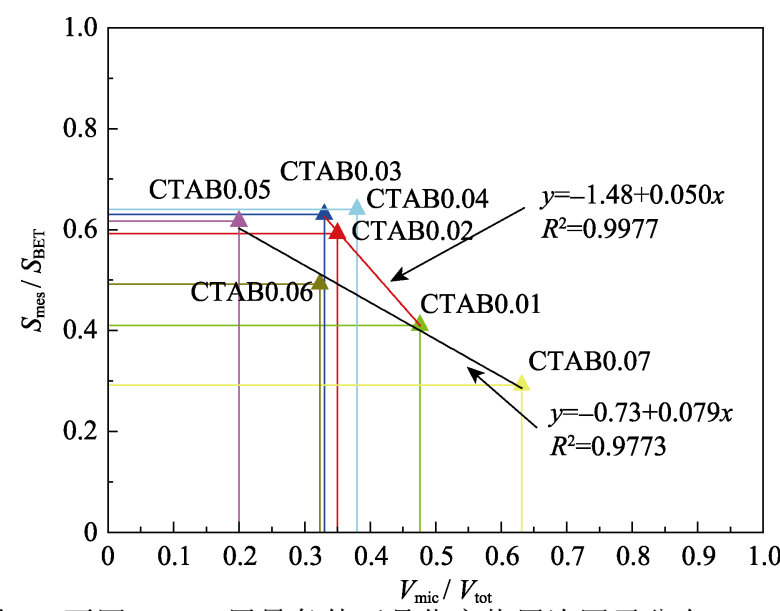

图 6 不同 CTAB 用量条件下晶化产物层次因子分布

Fig. 6 Hierarchy factor of crystalline products with different CTAB additions

\subsection{MB 吸附效果对比}

通过研究产物对 $\mathrm{MB}$ 的吸附效果分析分子篮的 孔道层次结构变化。在 $50.00 \mathrm{mg} / \mathrm{L} \mathrm{MB}$ 溶液中, 分 别加入 $0.10 \mathrm{~g}$ 不同 CTAB 用量下的晶化产物, 室温 吸附 $12 \mathrm{~h}$, 吸附结果如图 7 所示。吸附效率顺序为: CTAB $0.05>$ CTAB 0.06 $>$ CTAB 0.07 $>$ CTAB 0.04> CTAB0.03>CTAB0.02>CTAB0.01; 其中, CTAB0.05 样品的 $S_{\mathrm{BET}}$ 为 $432.02 \mathrm{~m}^{2} / \mathrm{g}$, 层次因子 $\mathrm{HF}$ 为 0.12 , 吸 附容量最高为 $366.45 \mathrm{mg} / \mathrm{g}$, 说明分子篎的比表面积 及孔道层次结构对吸附效果的影响显著, $S_{\mathrm{BET}}$ 越大, 介孔越发达, 吸附容量就越大, 证明介孔有利于大 分子物质的进入。相比而言, CTAB 用量为 $0.05 \mathrm{~g}$ 时, 产物的吸附过程除孔道层次结构作用外, 还取决于 (H)ZSM-11 分子篎具有的垂直孔道 ${ }^{[20]}$ 。因此, 在结 构导向剂 CTAB 作用下, 多级孔分子篮的晶体种 类、孔道结构层次变化显著影响吸附过程。

\section{3 结论}

1) Si-ATP 产物中存在 $\mathrm{Si}-\mathrm{O}_{4}$ 非对称结构和四面 体双环结构, 证明 Si-ATP 的骨架结构与分子篮的骨 架结构相似。

2) XRD 分析说明 CTAB 用量导致分子篮从 ZSM-5 型向(H)ZSM-11 型转化, SEM 结果显示粒径 的尺寸和形状发生不规则变化。

3) CTAB 用量为 $0.05 \mathrm{~g}$ 时, HF 值为 0.12 , 说明 (H)ZSM-11 分子篮存在以介孔为主的多级孔道; 研 究 HF 与 CTAB 用量之间的线性关系, 说明结构导 向剂 CTAB 导致分子䇻的孔道层次结构发生变化。

4) MB 吸附过程中, 证实分子篮的表面形态及 孔道层次结构显著影响其吸附效果, 且介孔有利于

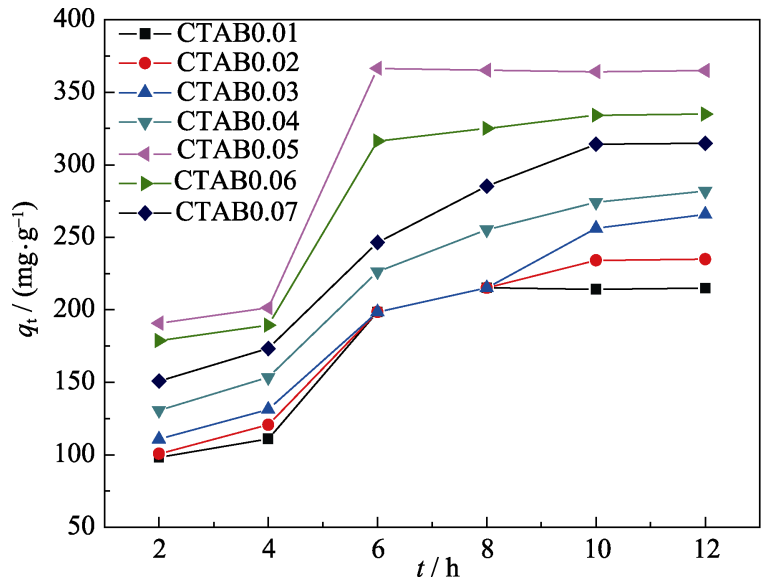

图 7 晶化产物对 $\mathrm{MB}$ 的吸附性能

Fig. 7 Adsorption properties of crystalline products to MB

大分子物质的进入。

\section{参考文献:}

[1] NA K, CHOI M, PARK W, et al. Pillared MFI zeolite nanosheets of a single-unit-cell thickness. Journal of the American Chemical Society, 2010, 132(12): 4169-4177.

[2] GUO YA-PING, WANG HAI-JIN, GUO YA-JUN, et al. Fabrication and characterization of hierarchical ZSM- 5 zeolites by using organosilanes as additives. Chemical Engineering Journal, 2011, 166(1): 391-400.

[3] SERRANO D P, SANZ R, PIZARRO P, et al. Synthesis of hierarchical TS-1 zeolite from silanized seeds. Topics in Catalysis, 2010, 53(19/20): 1319-1329.

[4] CUI SHENG-HANG, ZHANG JUN-TAO, SHEN ZHI-BING. Hierarchical ZSM-5 zeolite: synthesis and catalytic applications. Chemical Industry and Engineering Progress, 2015, 34(9): 33313336.

[5] TAO YOU-SHENG, KANOH H, ABRAMS L, et al. Mesoporemodified zeolites: preparation, characterization, and applications. Chemical Reviews, 2006, 37(21): 896-910.

[6] CHAL R, GERARDIN C, BULUT M, et al. Overview and industrial assessment of synthesis strategies towards zeolites with mesopores. ChemCatChem., 2011, 3(1): 67-81.

[7] DENG QIANG, ZHANG XIANG-WEN, WANG LI, et al. Catalytic isomerization and oligomerization of endo-dicyclopentadiene using alkali-treated hierarchical porous HZSM-5. Chemical Engineering Science, 2014, 135: 540-546.

[8] XU YI-QUAN, FANG YE-SEN. Classification and designation of clay minerals. Silicate Bulletin, 1982, 3(1): 3-10, 16.

[9] YANG DONG-HUA, LI JIAN-HUA, WANG XIN-BO, et al. Rapid synthesis and catalytic application of B-EU-1/ZSM-5 composite zeolite based on dual template. Journal of Inorganic Materials, 2016, 31(3): 248-256.

[10] LU LEI, LI XING-YANG, LIU XIAO-QIN, et al. Enhancing hydrostability and catalytic performance of metal-organic frameworks by hybridizing with attapulgite, a natural clay. J. Mater. Chem., 2015, 3(13): 6998-7005.

[11] ZHAO DENGSHAN, LI DENGHAO. Study on preparation of 4A zeolite molecular sieve from attapulgite clay. Non-Metallic Mines, 
2008, 31(6): 7-9.

[12] SHAO HUI, CHEN JING-JING, ZHONG JING, et al. Development of MeSAPO-5 molecular sieves from attapulgite for dehydration of carbohydrates. Industrial \& Engineering Chemistry Research, 2015, 54(5): 1470-1477.

[13] ZHENG SHU-QIN, GAN JIE, REN SHAO, et al. Synthesis of hierarchical catalytic materials from Si-Al gel and kaolin by hydrothermal crystallization. Acta Petroleisinica, 2014, 30(1): 32-37.

[14] ZHOU XIAO-ZHAO, LIU YAN, MENG XIANG-JU, et al. Synthesis and catalytic cracking performance of Fe/Ti-ZSM-5 zeolite from attapulgite mineral. Chinese Journal of Catalysis, 2013, 34(8): 1504-1512.

[15] FYFE C, LIN Z, TONG C, et al. Simple, efficient synthesis of zeolite ZSM-11 (MEL) at temperatures below $100^{\circ} \mathrm{C}$ using very dense gels. Microporous and Mesoporous Materials, 2012, 150(1): 7-13.

[16] RUTKOWSKA M, PACIA I, BASAG S, et al. Catalytic performance of commercial Cu-ZSM-5 zeolite modified by desilication in
$\mathrm{NH}_{3}-\mathrm{SCR}$ and $\mathrm{NH}_{3}-\mathrm{SCO}$ processes. Microporous and Mesoporous Materials, 2017, 246: 193-206.

[17] ZHANG KE, LIU YUN-QI, ZHAO JIN-ZHONG, et al. Hierarchical porous ZSM-5 zeolite synthesized by in situ zeolitization and its coke deposition resistance in aromatization reaction. Chinese $J$. Chem, 2012, 30(3): 597-603.

[18] THOMMES M, KANEKO K, NEIMARK A V, et al. Physisorption of gases, with special reference to the evaluation of surface area and pore size distribution (IUPAC Technical Report). Pure Appl. Chem., 2015, 87: 1051-1069.

[19] BIEMELT T, SELZER C, SCHMIDT F, et al. Hierarchical porous zeolite ZSM-58 derived by desilication and desilication reassembly. Microporous and Mesoporous Materials, 2014, 187(3): 114-124.

[20] YU QING-JU, CUI CHAOYUE, ZHANG QIANG, et al. Hierarchical ZSM-11 with intergrowth structures: synthesis, characterization and catalytic properties. Journal of Energy Chemistry, 2013, 22(5): 761-768. 